\title{
Giant Fibroids: Diagnosis and Management Modality: About Two Cases and Literature Review
}

Benayada Mariyam ${ }^{1 *}$, Ch'michi Nadia ${ }^{1}$, Essabbagh Youssef ${ }^{1}$, Echchikhi Meriam², Bennani Aicha ${ }^{2}$, Lakhdar Amina ${ }^{2}$, Zeraidi Najia ${ }^{2}$, Kharbach Aicha², Baidada Aziz ${ }^{2}$

${ }^{1}$ Gynaecology-Obstetrics and Endoscopy Department, Maternity Souissi, University Hospital Center IBN SINA, University Mohammed V, Rabat, Morocco

${ }^{2}$ Department of Medical Imaging and Radiodiagnosis, Children’s Hospital, CHU Ibn Sina, Rabat, Morocco

DOI: $10.36347 /$ sjams.2020.v08i10.019

| Received: 30.09.2020 | Accepted: 14.10.2020 | Published: 19.10.2020

*Corresponding author: Benayada Mariyam

\section{Abstract}

Review Article

Uterine fibroids are benign pathologies, very common in women of childbearing age. They are the first cause of hysterectomy in women before menopause. Giant forms are rare. It raises diagnostic difficulties with other rare uterine tumors such as leiomyosarcoma. Magnetic resonance imaging specifies the organ of origin, the volume and the main relationships of the fibromyoma with adjacent structures. Medical treatment and embolization facilitates surgical treatment. We report here two cases of women with giant fibroma who underwent hysterectomy at the SOUISSI maternity hospital in Rabat.

Keywords: Giant fibroids, Magnetic resonance imaging, hysterectomy.

Copyright $\odot 2020$ The Author(s): This is an open-access article distributed under the terms of the Creative Commons Attribution 4.0 International License (CC BY-NC 4.0) which permits unrestricted use, distribution, and reproduction in any medium for non-commercial use provided the original author and source are credited.

\section{INTRODUCTION}

Uterine leiomyomas are benign tumors that correspond to a degenerative proliferation of connective fibrous elements and smooth muscle tissue. It is the most common benign tumor in women of childbearing age. It affects 20 to $40 \%$ of women over 35 years of age $[1,2]$. These are benign tumors that lead either to diffuse fibromatosis or to the formation of multiple nodules, often numerous fibromyomas developed at the expense of smooth muscle fibers. They are hormonedependent tumors, and hyperestrogenism favors their growth. Uterine fibroma is characterized by its variable anatomical forms responsible for its clinical polymorphism. Therapeutic management depends on several parameters: age, hormonal status of patients, size, number, location of fibroids and desire for pregnancy.

In some rare cases, they can reach an enormous volume and be the cause of a mass effect and sometimes serious complications. In imaging, ultrasound is not specific, magnetic resonance imaging is more efficient. The diagnosis of certainty is histological after the surgical treatment. Medical treatment and embolization facilitates surgical treatment which must be as conservative as possible.

\section{Comment \\ Observation $\mathbf{n}^{\circ} \mathbf{1}$}

It is Mrs. F.S. 36 years old, single, a teacher by profession. She has no notable pathological antecedents and has been suffering from menometrorrhagia for more than 4 months, with abdominal volume increase associated with urinary disorders such as dysuria and digestive signs such as constipation, the whole evolving in a context of conservation of the general state of health. The clinical examination finds a patient conscious, with normal blood pressure: 12/6 mmhg, tachycardic with 102 beats /min, very pale and discolored conjunctiva. The vaginal touch combined with the abdominal palpation found a uterus very increased in volume reaching the xiphoid appendix with perception of a polylobed mass, regular, rounded, well limited, painless, and mobile, of firm consistency without associated adnexal masses.

The Pelvic ultrasonography shows a very large uterus with the presence of a myoma type 2-5 beyond the FIGO classification measuring $18 \mathrm{~cm}$; both ovaries seen are of normal size and no peritoneal effusion. A CT scan and an angioscan confirmed the diagnosis. After a sub-umbilical midline laparotomy, a hysterectomy was performed because the patient presented a very important hemorrhage uncontrolled by medical measures which allowed the removal of the 
uterus containing a huge myoma measuring $22 \mathrm{~cm}$ and weighing $2 \mathrm{~kg}$.

The Anatomopathological examination confirmed the necrobiosis of the myoma and ruled out any sign of malignancy.

The postoperative follow-up was simple, allowing for discharge on the fifth day (Figure $1 \& 2$ ).

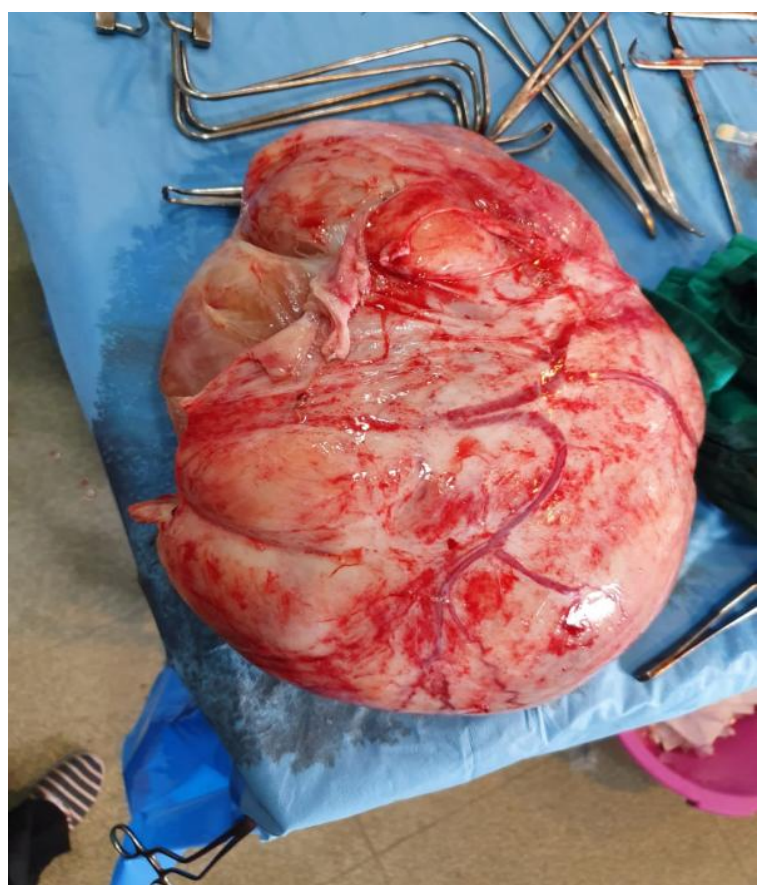

Fig-1: Piece of hysterectomy with the presence of a giant mass with anarchic vascularization

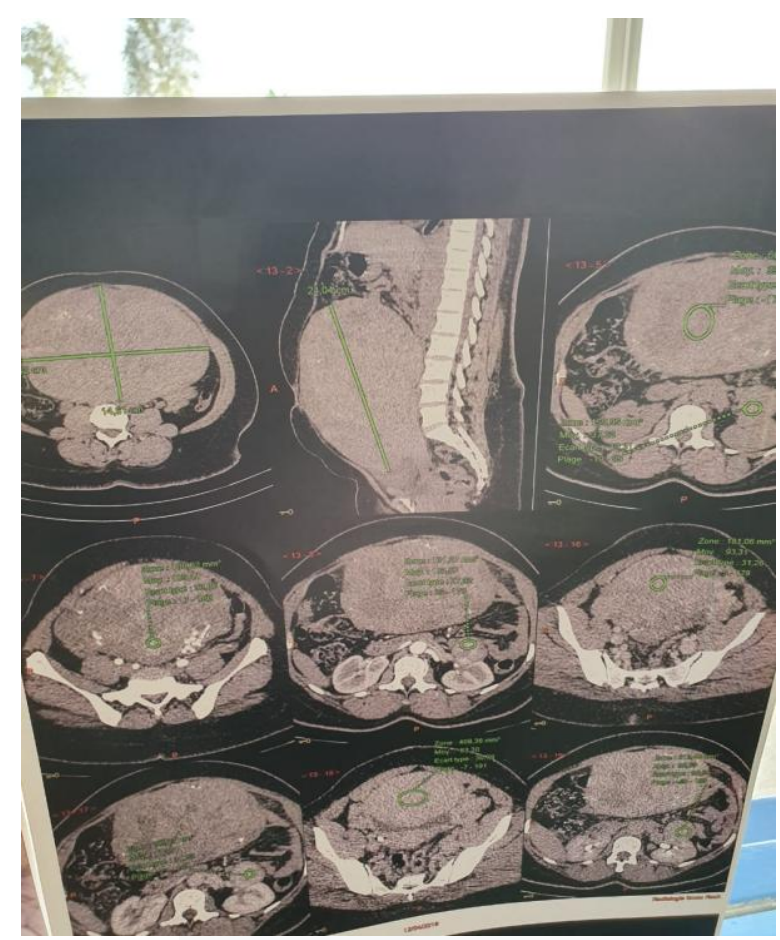

Fig-2: CT scan image shows a giant fibroid

\section{Observation $\mathbf{n}^{\circ} 2$}

This is a 52-year-old patient with no notable pathological antecedents, perimenopausal, G4P4, 4 vaginal deliveries, 4 live children. She consults for the sensation of increased abdominal volume complicated by a white line hernia, cycle disorders such as menorrhagia associated with pollakiuria, constipation and unquantified weight loss. The patient also reports the notion of asthenia and dyspnea.

The Clinical examination: Conscious patient, eupneic, BP: 13/7, HR: 86 bpm, conjunctiva are slightly discolored.

Vaginal + abdominal palping shows an enlarged uterus with the presence of a large mobile mass of painless irregular contours of firm consistency.

Abdominal ultrasound objectively shows an abdominal mass, however the exact origin (uterine or ovarian) could not be determined.

Surgery was performed. The Exploration showed that the mass was at the expense of the uterine fundus and measured $20 \mathrm{~cm}$.

The diagnosis of uterine fibroma was proposed and the patient was treated with a hysterectomy.

The diagnosis of uterine fibroma was confirmed after an anatomopathological study (Figure $3,4 \& 5)$.

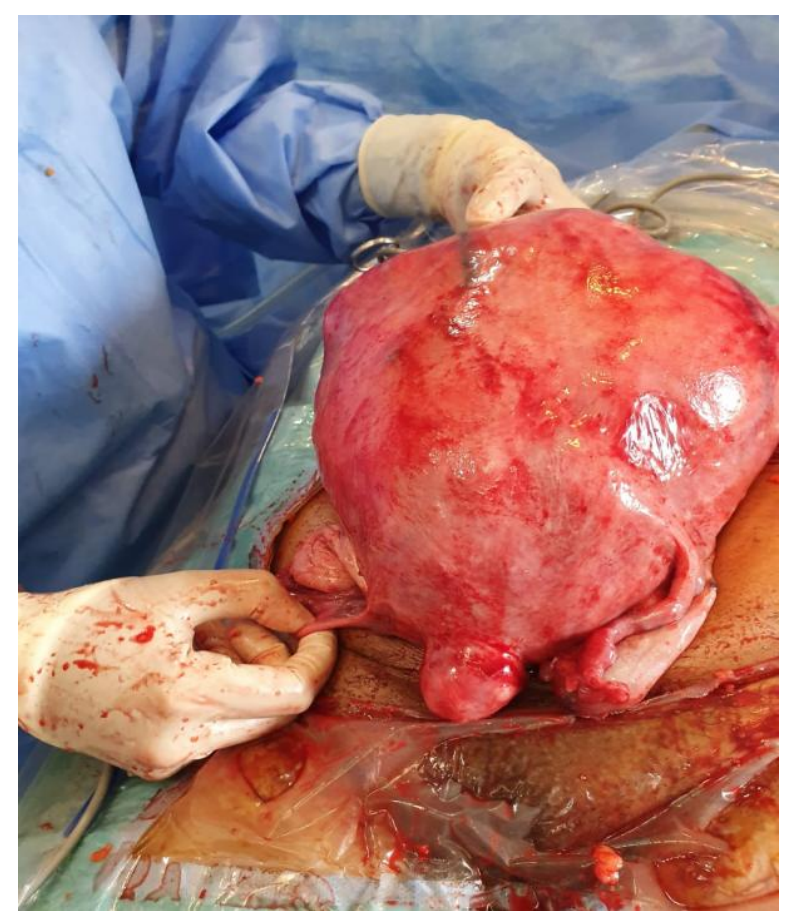

Fig-3: Uterus size greatly increased 


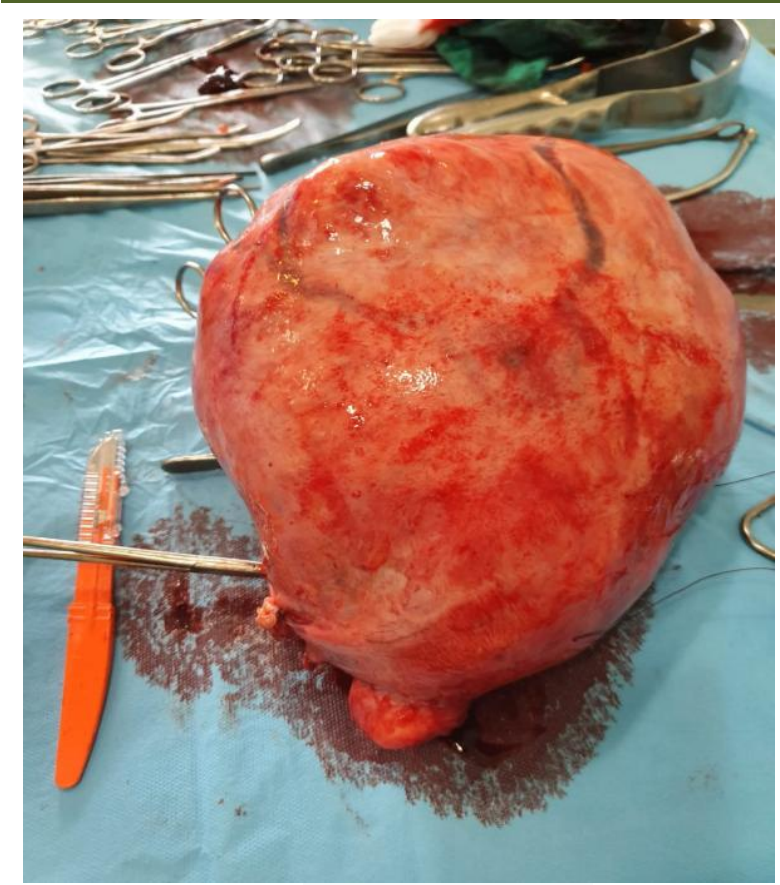

Fig-4: Hysterectomy piece

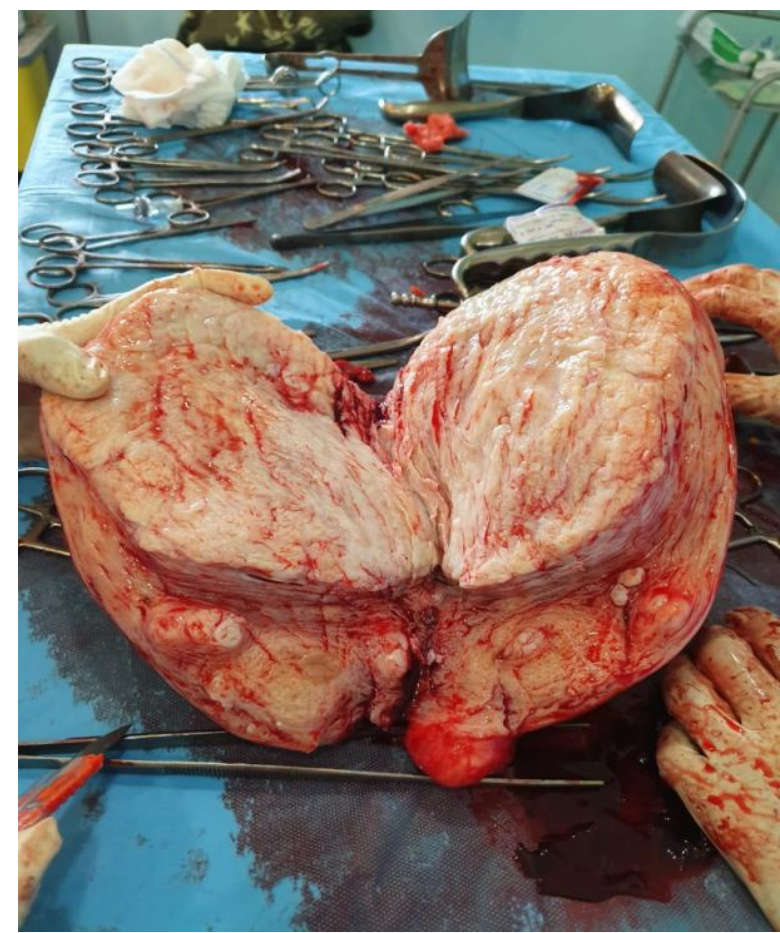

Fig-5: Presence of a giant fibroid and many small fibroids

\section{DISCUSSION}

The terms "fibroid", "myoma" and "leiomyoma" are all synonyms for the most common gynecologic tumors (their prevalence is between $70 \%$ and $80 \%$ in women who have reached the age of 50 years) [3].

Their prevalence increases with age and peaks in the forties. A study of hysterectomy found leiomyomas in $77 \%$ of uterine specimens [4]. In many women, myomas may be asymptomatic and are diagnosed incidentally through clinical examination or imaging analysis. However, myomas can also cause significant morbidity, including menstrual abnormalities (e.g. heavy, irregular and prolonged uterine bleeding), iron deficiency anemia, mass symptoms (e.g. pelvic pressure/pain, obstructive symptoms) and fertility problems. Symptomatic fibroids have a significant impact on women's quality of life and productivity [5].

Uterine fibroids are currently the most common indication worldwide for hysterectomy. In Canada, they account for $30 \%$ of all hysterectomies (with hysterectomy being the second most common surgery for women, after Caesarean section). Hysterectomy is associated with significant morbidity and mortality, and places a significant economic burden on the health care system [6,7]. The social and economic effects of uterine fibroids are therefore considerable.

Fibroids can reach large sizes and measure more than $11.5 \mathrm{~kg}$. Large sizes are rare and the clinical picture is noisy [8]. We then have a significant increase in abdominal volume with a mass effect due to bladder compression (pollakiuria, hydronephrosis or renal dysfunction), digestive symptoms (constipation, hypoxemia due to the decrease in stomach volume) [9], severe oedema, thrombosis, ulcer, calcified pelvic mass, hematimetry, severe pulmonary hypertension, and lifethreatening respiratory failure [10]. And in extreme cases, white line hernia due to significant abdominal distension. The torsion of the fibroid can mimic peritonitis. The large mass can degenerate rapidly, causing calcifications [11].

Ultrasonography (Trans abdominal, Trans vaginal, contrast hysteroecho) is the most widely used modality due to its availability, ease of use and costeffectiveness. This modality is particularly useful for evaluating the growth of myomas and adnexa [12]. Contrast ultrasonography (saline or gel) and hysteroecho (2D and 3D) are highly accurate diagnostic procedures that allow detection of sub-mucosal lesions (these modalities all have a sensitivity and specificity between $98 \%$ and $100 \%$ ) $[13,14]$. In women with large fibroids, diagnostic imaging occasionally reveals the presence of hydronephrosis (the clinical significance of this remains unknown). Complete ureteral obstruction is extremely rare [15].

Computed tomography has a limited value in delineating the location of myomas in relation to the endometrium or myometrium [16]. The magnetic resonance imaging is the most accurate modality for evaluating the adnexa [17] and uterus, providing information on the size, location, number, and perfusion of leiomyomas, as well as the presence of other uterine pathologies (such as adenomyosis and/or adenomyomas) $[18,19]$. 
The main differential diagnosis is uterine sarcoma, a rapidly evolving peri-menopausal mass that is difficult to explore on MRI and is also diagnosed histologically [9]. Giant fibroids can also mimic an ovarian carcinoma associated with a pseudo Meig's syndrome [10].

In the case of small fibroids, treatment is suggested only for complicated fibroids.In the case of large fibroids, medical and surgical treatment is necessary from the outset to improve patient comfort and reduce the compression caused by the mass.

The medical treatment is based on LHRH agonists in order to reduce the size before surgery and to facilitate the surgery. The surgical treatment depends on the patient's age, desire for pregnancy and height. It can be conservative in order to preserve the patient's fertility [20] or radical (i.e. hysterectomy). Embolization is currently under discussion. It would be interesting before surgery in cases of giant fibroids in order to reduce the size and facilitate surgery. Embolization by curaspon or gelatin is an interesting alternative. Indeed, it increases the chance of performing a conservative treatment such as a myomectomy with perfect enucleation and preserving the patient's fertility. It also allows for better operating conditions. Coelioscopy can even be performed with a reduction in duration and intraoperative bleeding. Complications are rare and very well tolerated by the patient $[21,22]$.

\section{Conclusion}

Uterine fibroma is a very common pathology in women of childbearing age. However, sometimes, in rare cases such as in our patient, it can reach enormous sizes and cause atypical symptoms and hinder the vital prognosis. For this reason, the diagnosis should not be overlooked. Some recent techniques are under discussion such as embolization to facilitate surgery and conservative treatment to preserve the patient's fertility.

\section{REFERENCE}

1. Bazot M, Salem C, Froment V, Chopier J. Bazot M, Salem C, Froment V, Chopier J. Pathologie myométriale. Encycl Méd Chir (Elsevier, Paris). 2002; 20:605-34.

2. Fernandez H, Gervaise A, Tyrac R. Fibrome utérin. Ency Med Chiru. 2002: 570-580.

3. Day Baird D, Dunson DB, Hill MC, Cousins D, Schectman JM. High cumulative incidence of uterine leiomyoma in black and white women: ultrasound evidence. Am J Obstet Gynecol. 2003; 188:100e7.

4. Cramer SF, Patel A. The frequency of uterine leiomyomas. Am J Clin Pathol. 1990; 94:435e8.

5. Vilos GA, Vilos A, Ferrazzi S. Symptomatic uterine fibroids (UFs) are common in premenopausal Canadian women and lead to reduced quality of life (QoL) across multiple domains. J Obstet Gynecol Can. 2014; 36(6 Suppl 1):S26.

6. Boyd LR, Novesky AP, Curtin JP. Effect of surgical volume on route of hysterectomy and short-term morbidity. Obstet Gynecol. 2010; 116:909e15.

7. Clark-Pearson DL, Geller EL. Complications of hysterectomy. Obstet Gynecol. 2013; 121:654e73.

8. Cissé M, Konaté I, Ka O, Dieng M, Dia A, Toure CT. Incarcerated procidentia due to giant cervical fibromyoma: a case report. Journal de gynecologie, obstetrique et biologie de la reproduction. $2008 \mathrm{Dec} ; 37(8): 802-803$.

9. Juglard R, Tourrette JH, Terrier JP, Dussaut JP, Barea D, Colineau X, Solacroup JC. Fibrome hydropique géant: à propos d'un cas. Journal de radiologie, 2002; 83(3): 372-374.

10. Mülayim B. Unaware of a large leiomyoma: A case report with respect to unusual symptoms of large leiomyomas. Annals of medicine and surgery. 2015 Dec 1;4(4):431-3.

11. Cole G, Rando C, Sibun L, Waldron T. Case report: A giant calcified uterus, likely due to benign leiomyoma. International journal of paleopathology. 2015 Sep 1;10:51-7.

12. Cantuaria GH, Anglioli R, Frost L, Duncan R, Penalver MA. Comparison of bimanual examination with ultrasound before hysterectomy for uterine leiomyoma. Obstet Gynecol. 1998; 92:109e12

13. Farquhar C, Ekeroma A, Furness S, Arroll B. A systematic review of transvaginal ultrasonography, sonohysterography and hysteroscopy for the investigation of abnormal uterine bleeding in premenopausal women. Acta Obstet Gynecol Scand. 2003; 82: 493e504.

14. Makris N, Kalmantis K, Startados N, Papadimitriou A, Mantzaris G, Antsaklis A. Three dimensional hysterosonography versus hysteroscopy for the detection of intracavitary uterine abnormalities. Int $\mathrm{J}$ Gynecol Obstet. 2007;95:6e9

15. Vercellini P, Crosignani PG, Mangioni C, Imparato E, Ferrari A, De Giorgi O. Treatment with a gonadotrophin releasing hormone agonist before hysterectomy for leiomyomas: results of a multicentre, randomized controlled trial. $\mathrm{Br} \mathrm{J}$ Obstet Gynaecol. 1998; 105:1148e54.

16. Bradley LD, Falcone T, Magen AB. Radiographic imaging techniques for the diagnosis of abnormal uterine bleeding. Obstet Gynecol Clin North Am. 2000; 27:245e76.

17. Adusumilli S, Hussain HK, Caoili EM, Weadock WJ, Murray JP, Johnson TD, Chen Q, Desjardins B. MRI of sonographically indeterminate adnexal masses. American journal of roentgenology. 2006 Sep;187(3):732-40.

18. Dueholm M, Lundorf E, Hansen ES, Ledertoug S, Olesen F. Accuracy of magnetic resonance 
imaging and transvaginal ultrasonography in the diagnosis, mapping, and measurement of uterine myomas. Am J Obstet Gynecol. 2002; 186:409e15.

19. Stamatopoulos CP, Mikos T, Grimbizis GF, Dimitriadis AS, Efstratiou I, Stamatopoulos P, Tarlatzis BC. Value of magnetic resonance imaging in diagnosis of adenomyosis and myomas of the uterus. Journal of minimally invasive gynecology. 2012 Sep 1;19(5):620-6.

20. Nguyen-Duc H. Volumineux fibrome utérin chez une adolescente de 15 ans: Myome géant à l'adolescence. Journal de gynécologie obstétrique et biologie de la reproduction. 2003;32(8):748-50.

21. Loffrody R, Ens Alem B, Guium B, Dranssajrmt L, Erais JP, Cercuedil K, Ause R. Embolisation préopératoire des volumineuxfibromesuterins de la femme jeune: suivi IRM à long terme.

22. Dumousset M, Cassagnes C, Mage B. Embolisation préopératoire des fibromesuterins (EPFU) DE LA FEMME JEUNE (22 PATIENTES) 